\title{
Increased Seizure Susceptibility in a Mouse with Diacylglycerol Kinase $\beta$ Deficiency
}

\author{
Mitsue Ishisaka $^{1}$, Kazuhiro Tsuruma ${ }^{1}$, Masamitsu Shimazawa ${ }^{1}$, Yasuhito Shirai ${ }^{2}$, Naoaki Saito ${ }^{3}$, \\ Hideaki Hara $^{1^{*}}$
}

${ }^{1}$ Department of Biofunctional Evaluation, Gifu Pharmaceutical University, Gifu, Japan; ${ }^{2}$ Graduate School of Agriculture, Kobe, Japan; ${ }^{3}$ Biosignal Research Center, Kobe University, Kobe, Japan.

Email: ${ }^{*}$ hidehara@gifu-pu.ac.jp

Received March $1^{\text {st }}, 2013$; revised April $3^{\text {rd }}, 2013$; accepted May $10^{\text {th }}, 2013$

Copyright (C) 2013 Mitsue Ishisaka et al. This is an open access article distributed under the Creative Commons Attribution License, which permits unrestricted use, distribution, and reproduction in any medium, provided the original work is properly cited.

\begin{abstract}
Diacylglycerol kinase (DGK) is an enzyme that converts diacylglycerol to phosphatidic acid. Several DGK isoforms have been implicated in the pathogenesis of seizure, but the role of DGK $\beta$ in seizure is unknown. In the present study, we investigated the involvement of DGK $\beta$ in seizure using DGK $\beta$ knockout (KO) mice. Seizures were more severe in DGK $\beta$ KO mice than in wild-type (WT) mice after pentylenetetrazol (PTZ) treatment and after kainic acid treatment, but there were no differences in latency to seizure. The expression levels of DGK $\beta$ in the hippocampal CA1, CA3, or DG areas did not differ between PTZ $(60 \mathrm{mg} / \mathrm{kg})$ treatment and saline treatment. There were fewer parvalbumin-positive interneurons in the hippocampal CA3 area in DGK $\beta$ KO mice than in control WT mice, which might partly account for the increased seizure susceptibility displayed by DGK $\beta$ KO mice. These results suggest that DGK $\beta$ may play a pivotal role in the development of the relevant interneurons, and that on inherent deficiency of DGK $\beta$ increases the animal's sensitivity to seizure-inducing stimuli.
\end{abstract}

Keywords: Diacylglycerol Kinase; Seizure; Parvalbumin

\section{Introduction}

The term "epilepsy" covers a common and diverse set of chronic neurological disorders, and in many cases, it is characterized by seizures. Various new antiepileptic drugs have been developed in the past decade, yet epilepsy remains resistant to drug therapy in about one-third of patients [1]. Because the biologic basis of such pharmacoresistance is multifactorial and because it varies from one patient to another, it is important to identify the precise cause of the problem.

Epilepsy has many underlying causes. It can be caused by an abnormality of brain tissues, such as a brain tumor or head injury, or by disruption of the balance between inhibitory and excitatory neurons in the brain. It has been reported that the excitability of principal hippocampal neurons increases with age, and also that more than a third of epileptic patients are over 65 years of age [2]. To judge from these reports, the inhibitory-excitatory balance among neurons may be important in epilepsy. On the other hand, genetic factors also play an important role,

"Corresponding author. and various mutations (for example, in ion channels) have been reported in epileptic individuals [3].

Diacylglycerol kinase (DGK) is an enzyme that converts diacylglycerol (DG) to phosphatidic acid (PA). To date, 10 isozymes have been identified and some isozymes have been reported to be involved in seizure conditions. For example, DGKe, which is classified as a type III DGK, is reported by associating with epileptogenesis and seizure susceptibility [4], while DGK $\zeta$, which is classified as a type IV DGK, is also involved in seizure susceptibility [5]. These reports actually suggest that DGKs may impede the development of seizure.

$\mathrm{DGK} \beta$ is classified as a type I DGK, and it possesses $\mathrm{Ca}^{2+}$-coordinating residues of two EF-hand motifs, cysteine residues of two cysteine-rich zinc-finger-like sequences, and a putative ATP-binding site, and is expressed in various brain regions, such as the olfactory bulb, cortex, striatum, and hippocampus [6]. Previously, we reported that $\operatorname{DGK} \beta$ plays a role in brain function and that $\mathrm{DGK} \beta$ knockout (KO) mice display various disorders of higher brain functions, such as cognitive impairment, hyperactivity, reduced anxiety, and careless be- 
havior [7-9].

In the present study, we investigated the seizure susceptibility in $\operatorname{DGK} \beta$ KO mice using pentylenetetrazol (PTZ) and kainic acid. Furthermore, we investigated the expression changes of DGK $\beta$ proteins during seizure by western blotting and immunostaining and the numbers of parvalbumin-positive interneurons.

\section{Material and Methods}

\subsection{Animals}

$\mathrm{DGK} \beta \mathrm{KO}$ mice $(\mathrm{C} 57 \mathrm{BL} / \mathrm{N})$ were generated using the Sleeping Beauty transposon system and mice were backcrossed for more than 9 generations, as described in our previous report [7]. Wild-type (WT) and DGK $\beta$ KO mice were generated by breeding heterozygous mutants, and we used male WT littermates as a control group for the male DGK $\beta$ KO mice. The animals (2- to 3-month old) were housed at $24^{\circ} \mathrm{C} \pm 2{ }^{\circ} \mathrm{C}$ under a $12 \mathrm{~h}$ light-dark cycle (lights on from 8:00 to 20:00), and they all had ad libitum access to food and water. All procedures relating to animal care and treatment conformed to the animal care guidelines of the Animal Experiment Committee of Gifu Pharmaceutical University. All efforts were made to minimize both suffering and the number of animals used.

\subsection{PTZ-Induced Seizure}

PTZ (Sigma Aldrich, St. Louis, MO, USA) dissolved in saline was injected intraperitoneally (i.p.) at a dose of 60 $\mathrm{mg} / \mathrm{kg}$. Animals were monitored for $30 \mathrm{~min}$ after the injection. Behavioral responses were recorded using a video camera and scored using the following scale: 0 , no abnormality; 1, exploring, sniffing, and grooming ceased, becoming motionless; 2 , head-nodding, facial and forelimb clonus; 3, myoclonic jerks of the head and neck, with brief twitching movements, or repetitive movements with head-bobbing or tail rigidity; 4 , forelimb or forelimb and hindlimb clonus, reciprocal forepaw padding, hindlimb abduction, continuous rearing, and falling, Straub tail response; 5 , tonic convulsions; 6 , death. Total seizure scores were calculated by summing up the minute-byminute scores.

\subsection{Kainic Acid-Induced Seizure}

Kainic acid (Sigma Aldrich, St. Louis, MO, USA) was dissolved in phosphate-buffered saline (PBS) and administered i.p. at a dose of $30 \mathrm{mg} / \mathrm{kg}$. Animals were monitored for $60 \mathrm{~min}$ after the injection. Behavioral responses were scored using the following scale: 0 , no response; 1, immobility and staring; 2 , forelimb and/or tail extension, rigid posture; 3 , repetitive movements, head-bobbing; 4, rearing and falling; 5, continuous rearing and falling; 6, severe clonic-tonic seizures; 7, death. Total seizure scores were calculated by summing up every-five-minute scores. The behavioral assessments described above were performed in a blind manner.

\subsection{Immunostaining}

For immunostaining studies, mice were injected with sodium pentobarbital $(50 \mathrm{mg} / \mathrm{kg}$, i.p.) (Nacalai Tesque, Kyoto, Japan) at 5 or 20 min after a PTZ injection then perfused with $4 \%$ paraformaldehyde in $0.1 \mathrm{M}$ phosphate buffer (PB; pH 7.4). Brains were removed after a 10-min perfusion fixation at $4^{\circ} \mathrm{C}$, then immersed in the same fixative solution overnight at $4^{\circ} \mathrm{C}$. They were then immersed in $25 \%$ sucrose in $0.1 \mathrm{M}$ PB for $24 \mathrm{~h}$, and embedded in a supporting medium for frozen-tissue specimens (OCT compound; Tissue-Teck). Cerebral sections $\left(14 \mu \mathrm{m}\right.$ thick) were cut on a cryostat at $-20^{\circ} \mathrm{C}$, and stored at $-80^{\circ} \mathrm{C}$ until staining. After washing twice with $0.01 \mathrm{M}$ PBS, sections were blocked with M.O.M. blocking reagent (M.O.M. immunodetection kit; Vector, CA, USA), then incubated with mouse anti-DGK $\beta$ antibody (1:200 dilution) for $72 \mathrm{~h}$ at $4^{\circ} \mathrm{C}$. They were washed with $0.01 \mathrm{M}$ PBS and then incubated for $1 \mathrm{~h}$ at room temperature with a mixture of Alexa Fluor $546 \mathrm{~F}\left(\mathrm{ab}^{\prime}\right)_{2}$ fragment of anti-mouse $\operatorname{IgG}(\mathrm{H}+\mathrm{L})$ (1:1000 dilution; Molecular Probes, OR, USA). Nuclei were stained with Hoechst 33,342 (1:1000 dilution; Invitrogen, Carlsbad, CA, USA). Images were obtained under a fluorescence microscope, at $\times 120$ magnification.

For the immunostaining of parvalbumin, cerebral sections $(10 \mu \mathrm{m}$ thick) were washed for $5 \mathrm{~min}$ in $0.01 \mathrm{M}$ PBS, then treated with $0.3 \%$ hydrogen peroxidase in $10 \%$ methanol. They were then washed three times in $0.01 \mathrm{M}$ PBS, followed by a 30-min pre-incubation with M.O.M. blocking reagent (M.O.M. immunodetection kit; Vector). Next, they were incubated with anti-parvalbumin antibody (1:1000 dilution; Millipore, Bedford, MA, USA) for $24 \mathrm{~h}$ at $4^{\circ} \mathrm{C}$. After a 15-min rinse in 3-change of 0.01 $\mathrm{M}$ PBS, the sections were incubated with biotinylated second antibody for $20 \mathrm{~min}$, and then with an avidinbiotin peroxidase complex for $30 \mathrm{~min}$ (both at room temperature). The number of parvalbumin-positive neuronal cell bodies was counted in a blind manner.

\subsection{Western Blotting}

For tissue sampling for western blotting, mice were decapitated at $20 \mathrm{~min}$ after a PTZ $(60 \mathrm{mg} / \mathrm{kg}$, i.p. $)$ or saline injection, and brains were quickly removed. The hippocampus was divided into CA1, CA3, and DG under a microscope. The experiment was performed as in a previous report [9]. For immunoblotting, the following primary antibodies were used: polyclonal anti-DGK $\beta$ (1:2000 dilution) and monoclonal anti- $\beta$-actin (1:5000 
dilution; Sigma Aldrich). HRP-conjugated goat antimouse IgG was used as the secondary antibody.

\subsection{Statistical Analysis}

Data are presented as mean \pm standard error of the mean (S.E.M.). Statistical comparisons were made by Student's $t$-test or Fisher's exact test (Vector, Tokyo, Japan). Probability (p) values of less than 5\% were considered statistically significant.

\section{Results}

\subsection{Diacylglycerol Kinase $\beta$ (DGK $\beta$ ) Knockout (KO) Mice Showed Severe Seizure after Pentylentetrazol (PTZ) Injection}

To investigate whether DGK $\beta$ KO mice exhibit normal or abnormal responses in drug-induced seizure models, we firstly used PTZ to induce seizure. PTZ, a gammaamino butyric acid $(\mathrm{GABA})_{\mathrm{A}}$-receptor antagonist, induces tonic-clonic seizure in mice. After receiving 60 $\mathrm{mg} / \mathrm{kg}$ PTZ, all WT and DGK $\beta$ KO mice displayed clonic convulsions (score 4), or else more serious seizures. About half the DGK $\beta$ KO mice displayed tonic convulsions (score $5 ; 5 / 9$ ), whereas almost all WT mice were allocated score 4 (only 1/10 was given score 5). We assessed changes over time in the seizure score (Figure 1(a)). In the WT mice, the seizure score peaked within 5 min and then slowly declined. On the other hand, in DGK $\beta$ KO mice the seizure score remained persistently

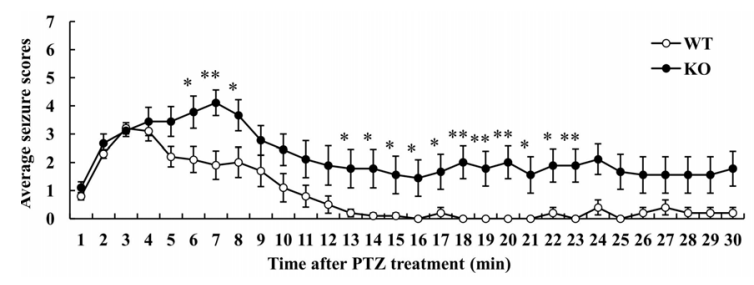

(a)

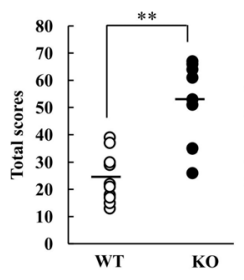

(b)

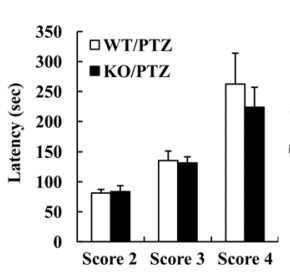

(c)

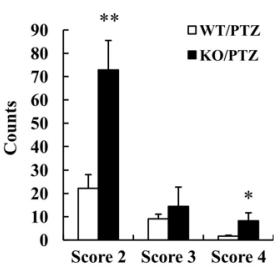

(d)
Figure 1. Behavioral changes in diacylglycerol kinase $\beta$ (DGK $\beta$ ) knockout (KO) mice after pentylenetetrazol (PTZ) treatment. (a) Mean seizure scores per one minute; (b) total seizure scores; (c) latency to reach a given seizure score; and (d) number of occurrences of a given seizure score, in WT and DGK $\beta$ KO mice after PTZ $(60 \mathrm{mg} / \mathrm{kg}$, i.p.) treatment. Values are expressed as the mean \pm S.E.M. (WT, $n=$ 10; KO, $\mathrm{n}=9$ ), ${ }^{*} \mathrm{p}<0.05 ;{ }^{* *} \mathrm{p}<0.01$ vs. WT mice (Student's $t$-test). high. In the analysis of the full $30 \mathrm{~min}$ of the study period, the total score was higher for DGK $\beta$ KO mice than for WT mice (Figure 1(b)). However, there was no difference between the mouse groups in the latency to seizure for scores 2, 3, or 4 (Figure 1(c)). The number of seizures scored 2 or 4 was greater for DGK $\beta$ KO mice than for WT mice (Figure 1(d)). These results suggest that DGK $\beta$ KO mice have an elevated sensitivity to PTZ.

\subsection{Diacylglycerol Kinase $\beta$ (DGK $\beta$ ) Knockout (KO) Mice Showed Severe Seizure after Kainic Acid Injection}

To investigate whether DGK $\beta$ KO mice might have abnormal susceptibility to another drug that induces seizure, we used kainic acid. This is an agonist for kainate-class ionotropic glutamate receptors, and is used to study the mechanisms underlying excitation-induced apoptosis and epilepsy. In the WT group, seizure activity increased slowly, with score 5 seizures being observed at $40 \mathrm{~min}$ after the injection (Figure 2(a)). The pattern was similars for DGK $\beta$ KO mice, but their seizure scores tended to be higher throughout the experiment (Figure 2(a)). The total seizure score for the full $60 \mathrm{~min}$ was higher for DGK $\beta$ KO mice than for WT mice (Figure 2(b)). However, there was no difference in the latency to seizure of score 3 between the two genotypes (Figure 2(c)). These results suggest that $\mathrm{DGK} \beta$ KO mice have a greater sensitivity than WT mice to kainic acid, too.

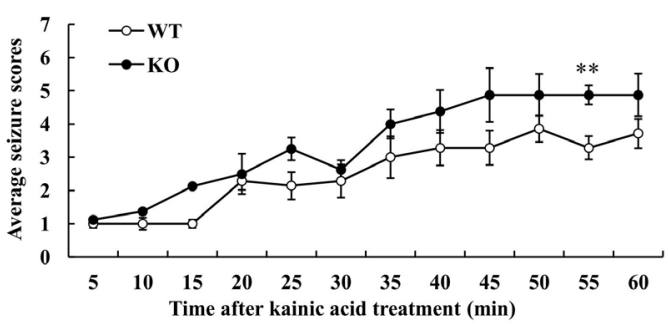

(a)

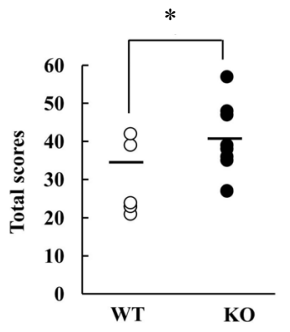

(b)

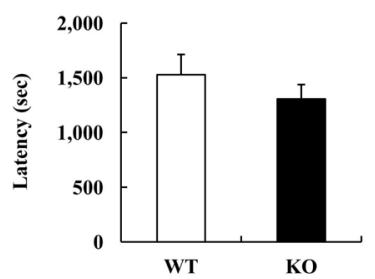

(c)
Figure 2. Behavioral changes in diacylglycerol kinase $\beta$ (DGK/) knockout (KO) mice after kainic acid treatment; (a) Mean seizure scores per five minute; (b) total seizure scores; and (c) latency to reach a given seizure score, in WT and DGK $\beta$ KO mice after kainic acid $(30 \mathrm{mg} / \mathrm{kg}$, i.p.) treatment. Values are expressed as the mean \pm S.E.M. (WT, $n=7$; KO, $\mathrm{n}=8),{ }^{*} \mathrm{p}<0.05 ;{ }^{* * *} \mathrm{p}<0.01$ vs. WT mice (Student's $t$-test). 


\subsection{The Expression Levels and Localization of Diacylglycerol Kinase $\beta($ DGK $\beta)$ after Pentylentetrazol (PTZ) Injection}

Previously, it was reported that in an animal model of transient ischemia, DGK $\zeta$ translocates rapidly from the nucleus to the cytoplasm of hippocampal neurons [10]. It has also been shown that upon reception of excitatory stimuli, DGK $\zeta$ changes its localization from the nucleus to the cytoplasm, and is degraded through the cytoplasmic ubiquitin-proteasome system, in the hippocampus [5]. We investigated whether the localization or expression level of DGK $\beta$ might change after PTZ treatment of the WT mice. However, we could detect no difference (vs. saline-injected mice) in the localization of $\mathrm{DGK} \beta$ protein at either 5 or $20 \mathrm{~min}$ after PTZ injection (data not shown). Investigation of the expression level of $\mathrm{DGK} \beta$ protein by western blotting likewise revealed no effect of PTZ injection (data not shown). These results suggest that DGK $\beta$ may have little relation to the development of seizure.

\subsection{Parvalbumin-Positive Cells Were Decreased in the Hippocampal CA3 Area in Diacylglycerol Kinase $\beta$ (DGK $\beta$ ) Knockout (KO) Mice}

Interneurons have been reported to play an important role in the control of seizure, and an imbalance between inhibitory interneurons and excitatory neurons can lead to various disease states [11-13]. Indeed, a decrease in the number of inhibitory interneurons causes an increase in seizure sensitivity [14]. We hypothesized that a decrement in inhibitory interneurons might contribute to the increment in seizure sensitivity observed here in DGK $\beta$ KO mice. In the hippocampal CA3 region, the number of parvalbumin-positive cells was reduced in DGK $\beta$ KO mice (vs. WT mice), but this was not the case in the CA1 region or dentate gyrus (Figure 3). In the cortex, there were no changes between WT and DGK $\beta$ KO mice (data not shown).

These results suggest that a decrement in inhibitory interneurons in CA3 might contribute to the increased seizure sensitivity observed in DGK $\beta$ KO mice.

\section{Discussion}

Previous studies have progressively clarified the localization of $\operatorname{DGK} \beta$ within the brain. $\operatorname{DGK} \beta$ is localized in neurons and its expression has been detected in the olfactory bulb, hippocampus, cortex, and striatum $[6,7]$. Within the hippocampus, DGK $\beta$ is expressed in the stratum oriens and stratum radiatum of CA1, CA2, and CA3 (not only in projection neurons, but also in interneurons) [15].

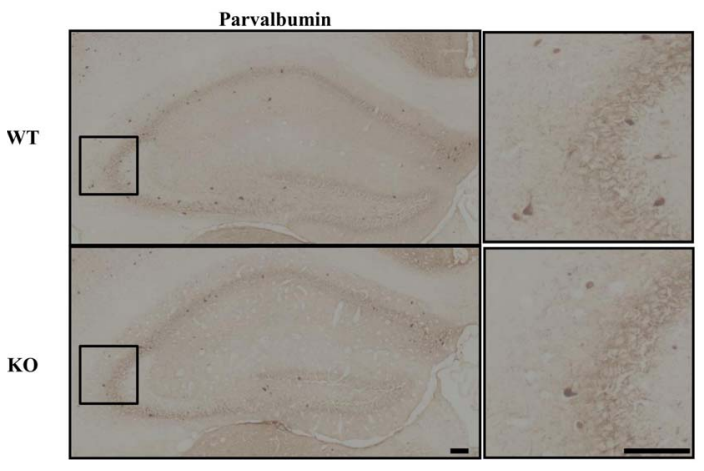

(a)

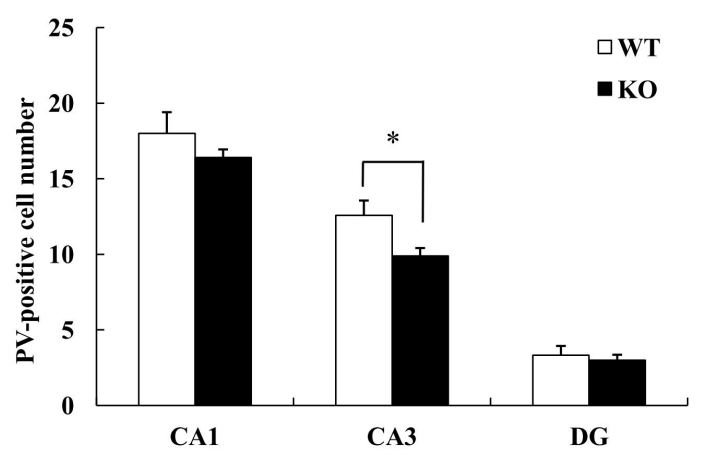

(b)

Figure 3. Parvalbumin-positive interneurons in hippocampal subregions. (a) Immunostaining for parvalbumin in the hippocampus of age-matched WT and DGK $\beta$ KO mice. Right panels show enlargements of the hippocampal CA3 region (shown by the box in the corresponding left panel). Scale bars $=100 \mu \mathrm{m}$; (b) Parvalbumin-positive cell numbers in the hippocampal subregions in WT and DGK $\beta$ KO mice. Values are expressed as the mean \pm S.E.M. (WT, $n=6$; KO, $\mathrm{n}=5$ ), ${ }^{*} \mathbf{p}<0.05$ vs. WT mice (Student's $t$-test).

Since DGK $\beta$ KO mice displayed more severe reactions to a given chemoconvulsant than their WT controls, we investigated whether they displayed abnormal localizations or expression levels. In a previous report, the expression levels and localization of $\mathrm{DGK}_{l}$, which is thought to be involved in seizure, did not relate to the animal's seizure status. However, DGK $\zeta$ was found to be translocated from nucleus to cytoplasm in response to chemoconvulsants, and a deficiency of DGK $\zeta$ led to an increased sensitivity to chemoconvulsants [5]. To judge from our results, the localization of $\mathrm{DGK} \beta$ is unchanged, and it remains at the cell surface, after PTZ treatment, indicating that $\mathrm{DGK} \beta$ may be a non-responsive protein during seizure in mice.

The interneurons in the hippocampus are GABAexpressing non-principal neurons, and the inhibitory input from various subpopulations of GABAergic interneurons to the principal neurons in different subfields of the hippocampus is necessary for the maintenance of network stability [16] Interneurons positive for the 
calcium-binding protein parvalbumin help to synchronize the hippocampal-network oscillations [17]. Furthermore, parvalbumin deficiency results in an increased susceptibility to seizure $[14,18-20]$. In the hippocampal CA3 region of $\mathrm{DGK} \beta \mathrm{KO}$ mice, the number of parvalbumin-positive cells was decreased (vs. WT controls), which would increase the susceptibility of DGK $\beta$ KO mice to seizure. Interestingly, the number of parvalbumin positive interneuron is decreased in the hippocampus of patients with bipolar disorder, too $[11,21,22]$. We reported ealier that DGK $\beta$ KO mice display mania-like behavior [8], and others have reported that a splice variant at the $\mathrm{COOH}$-terminal of $\mathrm{DGK} \beta$ is related to bipolar disorder [23]. In rat brain, the expression levels of DGK $\beta$ protein are reportedly slightly increased on postnatal day 7 , and increase rapidly between postnatal days 14 and 28 [24]. Around the same time as DGK $\beta$ expression increases, parvalbumin mRNA can be detected in the cereberal cortex and hippocampus [25]. These results may suggest that DGK $\beta$ regulates the development of parvalbumin-positive interneurons.

In conclusion, DGK $\beta$ KO mice display increased seizure susceptibility, which may be due to a loss of parvalbumin-positive interneurons in the CA3 hippocampal region. On the basis of such data, it is likely that $\mathrm{DGK} \beta$ plays a pivotal role in the development of such interneurons, while an inherent deficiency in DGK $\beta$ increases the animal's sensitivity to seizure-inducing stimuli.

\section{REFERENCES}

[1] A. M. Siegel, "Presurgical Evaluation and Surgical Treatment of Medically Refractory Epilepsy," Neurosurgical Review, Vol. 27, No. 1, 2004, pp. 1-18. doi:10.1007/s10143-003-0305-6

[2] H. Wallace, S. Shorvon and R. Tallis, "Age-Specific Incidence and Prevalence Rates of Treated Epilepsy in an Unselected Population of 2,052,922 and Age-Specific Fertility Rates of Women with Epilepsy," Lancet, Vol. 352, No. 9145, 1998, pp. 1970-1973. doi:10.1016/S0140-6736(98)04512-7

[3] C. A. Reid, S. F. Berkovic and S. Petrou, "Mechanisms of Human Inherited Epilepsies," Progress in Neurobiology, Vol. 87, No. 1, 2009, pp. 41-57. doi:10.1016/j.pneurobio.2008.09.016

[4] E. B. Rodriguez de Turco, W. Tang, M. K. Topham, F. Sakane, V. L. Marcheselli, C. Chen, A. Taketomi, S. M. Prescott and N. G. Bazan, "Diacylglycerol Kinase Epsilon Regulates Seizure Susceptibility and Long-Term Potentiation through Arachidonoyl-Inositol Lipid Signaling," The Proceedings of the National Academy of Sciences, Vol. 98, No. 8, 2001, pp. 4740-4745. doi:10.1073/pnas.081536298

[5] M. Okada, Y. Hozumi, T. Tanaka, Y. Suzuki, M. Yanagida, Y. Araki, C. Evangelisti, H. Yagisawa, M. K. Topham, A. M. Martelli and K. Goto, "DGKzeta Is De- graded through the Cytoplasmic Ubiquitin-Proteasome System under Excitotoxic Conditions, Which Causes Neuronal Apoptosis Because of Aberrant Cell Cycle Reentry," Cell Signalling, Vol. 24, No. 8, 2012, pp. 15731582. doi:10.1016/i.cellsig.2012.03.021

[6] K. Goto and H. Kondo, "Molecular Cloning and Expression of a $90-\mathrm{kDa}$ Diacylglycerol Kinase That Predominantly Localizes in Neurons," The Proceedings of the $\mathrm{Na}$ tional Academy of Sciences, Vol. 90, No. 16, 1993, pp. 7598-7602. doi:10.1073/pnas.90.16.7598

[7] Y. Shirai, T. Kouzuki, K. Kakefuda, S. Moriguchi, A. Oyagi, K. Horie, S. Y. Morita, M. Shimazawa, K. Fukunaga, J. Takeda, N. Saito and H. Hara, "Essential Role of Neuron-Enriched Diacylglycerol Kinase (DGK), DGKbeta in Neurite Spine Formation, Contributing to Cognitive Function," PLoS One, Vol. 5, No. 7, 2010, Article ID: e11602. doi:10.1371/journal.pone.0011602

[8] K. Kakefuda, A. Oyagi, M. Ishisaka, K. Tsuruma, M. Shimazawa, K. Yokota, Y. Shirai, K. Horie, N. Saito, J. Takeda and H. Hara, "Diacylglycerol Kinase Beta Knockout Mice Exhibit Lithium-Sensitive Behavioral Abnormalities," PLoS One, Vol. 5, No. 10, 2011, Article ID: e13447. doi:10.1371/journal.pone.0013447

[9] M. Ishisaka, K. Kakefuda, A. Oyagi, Y. Ono, K. Tsuruma, M. Shimazawa, K. Kitaichi and H. Hara, "Diacylglycerol Kinase Beta Knockout Mice Exhibit Attention-Deficit Behavior and an Abnormal Response on Methylphenidate-Induced Hyperactivity," PLoS One, Vol. 7, No. 5, 2012, Article ID: e37058. doi:10.1371/journal.pone.0037058

[10] H. Ali, T. Nakano, S. Saino-Saito, Y. Hozumi, Y. Katagiri, H. Kamii, S. Sato, T. Kayama, H. Kondo and K. Goto, "Selective Translocation of Diacylglycerol Kinase Zeta in Hippocampal Neurons under Transient Forebrain Ischemia," Neuroscience Letters, Vol. 372, No. 3, 2004, pp. 190-195. doi:10.1016/j.neulet.2004.09.052

[11] A. Y. Wang, K. M. Lohmann, C. K. Yang, E. I. Zimmerman, H. Pantazopoulos, N. Herring, S. Berretta, S. Heckers and C. Konradi, "Bipolar Disorder Type 1 and Schizophrenia Are Accompanied by Decreased Density of Parvalbumin- and Somatostatin-Positive Interneurons in the Parahippocampal Region," Acta Neuropathologica, Vol. 122, No. 5, 2011, pp. 615-626. doi:10.1007/s00401-011-0881-4

[12] Z. Q. Zhu, D. L. Armstrong, W. J. Hamilton and R. G. Grossman, "Disproportionate Loss of CA4 ParvalbuminImmunoreactive Interneurons in Patients with Ammon's Horn Sclerosis," Journal of Neuropathology \& Experimental Neurology, Vol. 56, No. 9, 1997, pp. 988-998. doi:10.1097/00005072-199709000-00004

[13] P. Marco, R. G. Sola, S. Ramon y Cajal and J. DeFelipe, "Loss of Inhibitory Synapses on the Soma and Axon Initial Segment of Pyramidal Cells in Human Epileptic Peritumoural Neocortex: Implications for Epilepsy," Brain Research Bulletin, Vol. 44, No. 1, 1997, pp. 47-66. doi:10.1016/S0361-9230(97)00090-7

[14] J. C. Gant, O. Thibault, E. M. Blalock, J. Yang, A. Bachstetter, J. Kotick, P. E. Schauwecker, K. F. Hauser, G. M. Smith, R. Mervis, Y. Li and G. N. Barnes, "Decreased Number of Interneurons and Increased Seizures in Neu- 
ropilin 2 Deficient Mice: Implications for Autism and Epilepsy," Epilepsia, Vol. 50, No. 4, 2009, pp. 629-645. doi:10.1111/j.1528-1167.2008.01725.x

[15] Y. Hozumi, M. Watanabe, K. Otani and K. Goto, "Diacylglycerol Kinase Beta Promotes Dendritic Outgrowth and Spine Maturation in Developing Hippocampal Neurons," BMC Neuroscience, Vol. 10, 2009, p. 99.

[16] T. F. Freund and G. Buzsaki, "Interneurons of the Hippocampus," Hippocampus, Vol. 6, No. 4, 1996, pp. 347470.

doi:10.1002/(SICI)1098-1063(1996)6:4<347::AID-HIPO 1>3.0.CO;2-I

[17] T. Klausberger, L. F. Marton, J. O’Neill, J. H. Huck, Y. Dalezios, P. Fuentealba, W. Y. Suen, E. Papp, T. Kaneko, M. Watanabe, J. Csicsvari and P. Somogyi, "Complementary Roles of Cholecystokinin- and Parvalbumin-Expressing GABAergic Neurons in Hippocampal Network Oscillations," The Journal of Neuroscience, Vol. 25, No. 42, 2005, pp. 9782-9793. doi:10.1523/JNEUROSCI.3269-05.2005

[18] B. Schwaller, I. V. Tetko, P. Tandon, D. C. Silveira, M. Vreugdenhil, T. Henzi, M. C. Potier, M. R. Celio and A. E. Villa, "Parvalbumin Deficiency Affects Network Properties Resulting in Increased Susceptibility to Epileptic Seizures," Molecular and Cellular Neuroscience, Vol. 25, No. 4, 2004, pp. 650-663. doi:10.1016/j.men.2003.12.006

[19] A. Andrioli, L. Alonso-Nanclares, J. I. Arellano and J. De Felipe, "Quantitative Analysis of Parvalbumin-Immunoreactive Cells in the Human Epileptic Hippocampus," Neuroscience, Vol. 149, No. 1, 2007, pp. 131-143. doi:10.1016/j.neuroscience.2007.07.029

[20] R. Kuruba, B. Hattiangady, V. K. Parihar, B. Shuai and A. K. Shetty, "Differential Susceptibility of Interneurons
Expressing Neuropeptide Y or Parvalbumin in the Aged Hippocampus to Acute Seizure Activity," PLoS One, Vol. 6, No. 9, 2011, Article ID: e24493. doi:10.1371/journal.pone.0024493

[21] H. Pantazopoulos, N. Lange, R. J. Baldessarini and S. Berretta, "Parvalbumin Neurons in the Entorhinal Cortex of Subjects Diagnosed with Bipolar Disorder or Schizophrenia," Biological Psychiatry, Vol. 61, No. 5, 2007, pp. 640-652. doi:10.1016/j.biopsych.2006.04.026

[22] C. Konradi, E. I. Zimmerman, C. K. Yang, K. M. Lohmann, P. Gresch, H. Pantazopoulos, S. Berretta and S. Heckers, "Hippocampal Interneurons in Bipolar Disorder," Arch Gen Psychiatry, Vol. 68, No. 4, 2011, pp. 340-350. doi:10.1001/archgenpsychiatry.2010.175

[23] A. Caricasole, E. Bettini, C. Sala, R. Roncarati, N. Kobayashi, F. Caldara, K. Goto and G. C. Terstappen, "Molecular Cloning and Characterization of the Human Diacylglycerol Kinase Beta (DGKbeta) Gene: Alternative Splicing Generates DGKbeta Isotypes with Different Properties," The Journal of Biological Chemistry, Vol. 277, No. 7, 2002, pp. 4790-4796. doi:10.1074/jbc.M110249200

[24] N. Adachi, M. Oyasu, T. Taniguchi, Y. Yamaguchi, R. Takenaka, Y. Shirai and N. Saito, "Immunocytochemical Localization of a Neuron-Specific Diacylglycerol Kinase Beta and Gamma in the Developing Rat Brain," Brain Molecular Brain Research, Vol. 139, No. 2, 2005, pp. 288-299. doi:10.1016/j.molbrainres.2005.06.007

[25] L. de Lecea, J. A. del Rio and E. Soriano, "Developmental Expression of Parvalbumin mRNA in the Cerebral Cortex and Hippocampus of the Rat," Molecular Brain Research, Vol. 32, No. 1, 1995, pp. 1-13. doi:10.1016/0169-328X(95)00056-X 